\title{
Description de deux nouvelles espèces \\ du genre Orientostrongylus Durette-Desset, 1971 \\ (Nématode, Héligmosome) \\ chez un Tupaïné et un Petauristiné de Malaisie
}

\author{
par M.-CI. DURETTE-DESSET et LIM BOO LIAT
}

Laboratoire de Zoologie (Vers) associé au C.N.R.S. ( $\mathrm{P}^{r}$ A.-G. CHabaud),

Muséum national d'Histoire naturelle, 43, rue Cuvier, F 75231 Paris Cedex 05

et Department of Ecology Medical, Institute of Medical Research, Kuala-Lumpur, Malaisie

\section{Résumé.}

- Orientostrongylus dendrogali n.sp., parasite de Dendrogale melanura (Tupaiiné), proche de $O$. brevispicularis (Singh, 1962), parasite de Rattus norvegicus en Inde, s'en distingue par la naissance des côtes externo-dorsales à la racine de la côte dorsale et la division de cette dernière dans son tiers antérieur.

- Orientostrongylus krishnansamyi n. sp., parasite d'Iomys horsfieldi (Petauristiné), proche de O. tenorai Durette-Desset, 1971, parasite de Muridés orientaux, s'en distingue par des spicules à pointe simple et un nombre plus élevé d'arêtes cuticulaires.

La présence d'un Orientostrongylus chez un Tupaiidé, animal relique, nous paraît un élément important pour confirmer la place de ce genre à la base de la seconde branche évolutive de la lignée Heligmonella.

\section{Summary.}

Description of two new species of the genus Orientostrongylus Durette-Desset, 1971 (Nematoda, Heligmosomidae) from a Tupaïnae and a Petauristinae in Malaysia.

- Orientostrongylus dendrogali n. sp., parasite of Dendrogale melanura (Tupaïnae) is closely related to $O$. brevispicularis (Singh, 1962) parasite of Rattus norvegicus in India. 
It can be separated from it by the externodorsal ribs which originate at the proximal end of the dorsal rib and by the division of that in its anterior third.

- Orientostrongylus krishnansamyi n.sp. parasite of Iomys horsfieldi (Petauristinae) is closely related to O. tenorai Durette-Desset, 1971, parasite of oriental Muridae. It can be differentiated from it by the spicules with a simple tip and by more numerous cuticular ridges.

The presence of an Orientostrongylus in a Tupaïdae, a relict animal, seems to be an important argument to confirm the status of this genus which is situated to the basis of the second evolution branch of the line Heligmonella.

Nous poursuivons dans cette note l'étude de l'importante collection de Nématodes Trichostrongyles déposée dans les collections du Département d'Ecologie Médicale de l'Institut de Recherche Médicale de Kuala-Lumpur. Les types sont déposés dans les collections du Muséum national d'Histoire naturelle de Paris.

\section{Orientostrongylus dendrogali n. sp.}

MATÉRIEL : $1 \delta^{\star}$ holotype, $1 q$ allotype $\left(n^{\circ} 756 \mathrm{HA}\right)$; nombreux $\delta^{\star}$ et $q$ paratypes (n 2 810).

Hôte: Dendrogale melanura (Thomas), $\mathrm{n}^{\circ} 2810$.

LOCALISATION: Intestin grêle.

Origine géographiQue: Mount Kinabalu, Bundu Tahan Reserve, 4200 pieds, Sabah, Nord Borneo. Lat. $116^{\circ} 30^{\prime} \mathrm{N}$, Long. 6 ${ }^{\circ}$ '.

\section{Description.}

Très petits Nématodes enroulés de façon senestre le long de leur ligne ventrale.

SYNLOPHE: de type Orientostrongylus avec 14 arêtes cuticulaires qui s'étendent tout le long du corps du bord de la vésicule céphalique jusqu'en avant de la bourse caudale chez le mâle et de la vulve chez la femelle (fig. 1, B).

MÂle: corps long de 3,2 mm, large de $60 \mu$ dans sa partie moyenne. Vésicule céphalique haute de $50 \mu$ sur $30 \mu$ de large. Anneau nerveux, pore excréteur et deirides situés respectivement à $155 \mu, 250 \mu$ et $270 \mu$ de l'apex. Esophage long de $325 \mu$ (fig. 1, A).

Bourse caudale légèrement asymétrique avec un lobe gauche plus développé. Disposition des côtes figurées en 1, D. Côte dorsale épaisse, divisée en 2 rameaux à son tiers inférieur. Côtes 8 naissant au tiers de la hauteur du tronc dorsal. Spicules longs de $120 \mu$. Leur partie postérieure est fine et ailée. La partie antérieure est élar- 
gie (fig. 1, E). Gubernaculum non vu. Cône génital très peu développé. Papilles zéro et 7 arrondies (fig. 1, D).
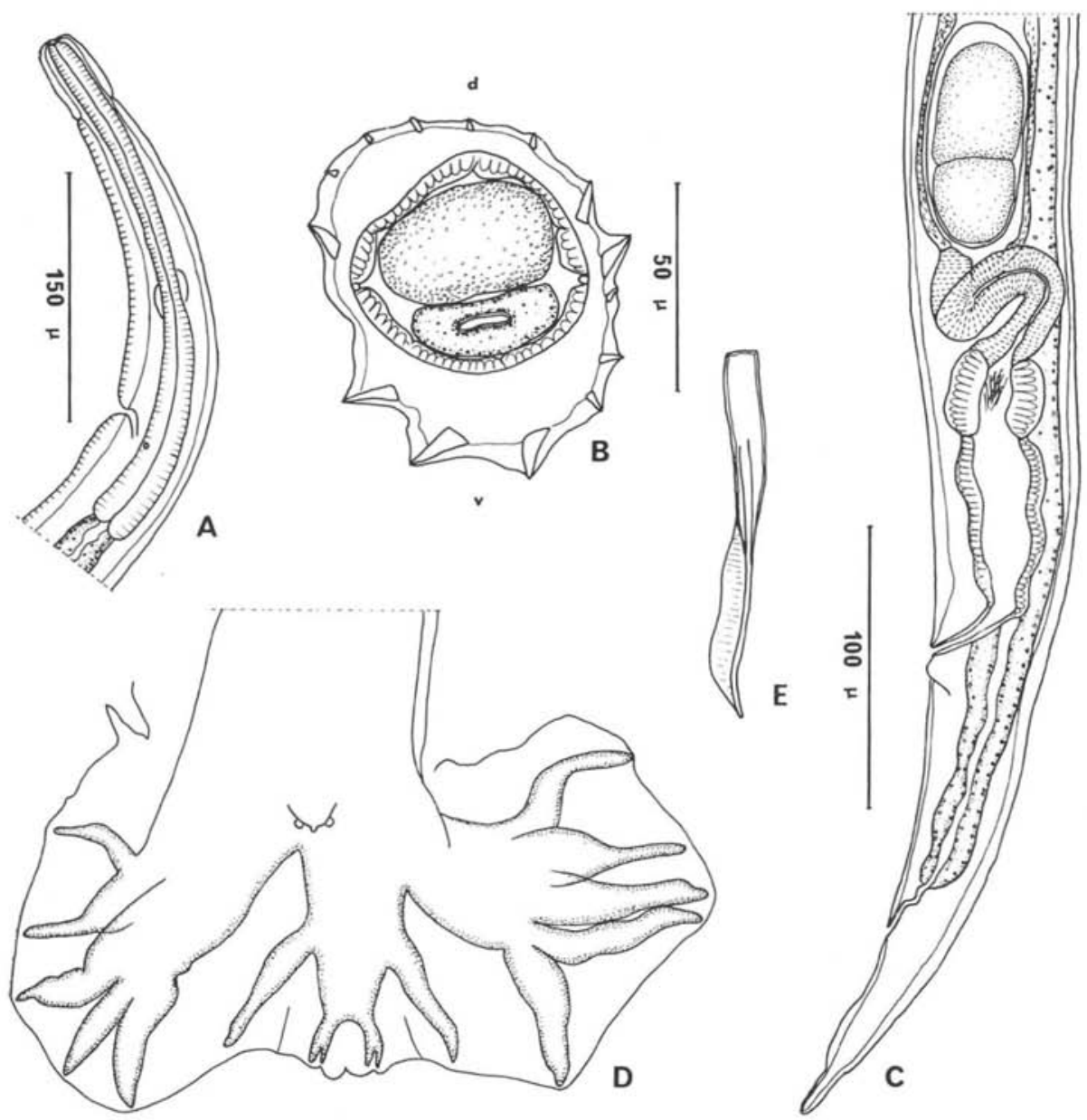

Fig. 1. - Orientostrongylus dendrogali n.sp. A, ô, extrémité antérieure, vue latérale gauche. $\mathrm{B}, \hat{\delta}$, coupe transversale au milieu du corps. $\mathrm{C}$, + , extrémité postérieure, vue latérale gauche. $\mathrm{D}, \hat{\delta}$, bourse caudale, vue ventrale. $\mathrm{E}$, spicule disséqué.

A, éch. : $150 \mu ; \mathrm{B}$, éch. : $50 \mu$; C, D, E, éch. : $100 \mu$.

Femelle : corps long de $3 \mathrm{~mm}$, large de $60 \mu$ dans sa partie moyenne. Vésicule céphalique haute de $50 \mu$ sur $30 \mu$ de large. Anneau nerveux, pore excréteur et deirides situés respectivement à $150 \mu, 220 \mu$ et $240 \mu$ de l'apex. Esophage long de $340 \mu$. La vulve s'ouvre à $210 \mu$ de la pointe caudale. Vagina vera, vestibule, sphincter 
et trompe longs respectivement de $28 \mu, 60 \mu, 25 \mu$ et $95 \mu$. Utérus long de $520 \mu$, contenant 8 œufs hauts de $72 \mu$ sur $30 \mu$ de large (fig. 1, C).

Queue fine, longue de $65 \mu$ (fig. 1, C).

\section{Discussion.}

Par les caractères de son synlophe (arêtes en nombre moyen, peu développées et sans gradient de taille défini), de sa bourse caudale (côte dorsale épaisse) et ses spicules courts, les spécimens du Dendrogale se rangent aisément dans le genre Orientostrongylus Durette-Desset, 1971.

Trois espèces sont actuellement décrites chez les Muridés et les Cricétidés orientaux : O. tenorai Durette-Desset, 1970, O. chinensis (Erhardova, 1959) et O. brevispicularis (K. S. Singh, 1962). La première espèce se différencie aisément par des spicules à pointe complexe et la seconde par son synlophe formé d'arêtes et de crêtes. Chez la $3^{\circ}$ espèce, les spicules sont aussi à pointe simple comme chez nos spécimens. Bien que la comparaison soit difficile à faire, car l'auteur n'a pas étalé la bourse caudale, nous notons cependant les différences suivantes: chez $O$. brevispicularis, les côtes externo-dorsales naissent à la racine de la côte dorsale et cette dernière est divisée dans son tiers antérieur. De plus, la distance anus-vulve, pour une longueur du corps plus grande, est un tiers plus petite. Le synlophe n'étant pas connu, et ces quelques différences étant appréciables, il nous semble préférable de ne pas identifier nos spécimens à ceux de Singh et de les nommer Orientostrongylus dendrogali n. sp.

\section{Orientostrongylus krishnansamyi n. sp.}

Matériel : $1 \delta^{\dagger}$ holotype, $1 q$ allotype ( ${ }^{\circ} 757$ HA), nombreux $\delta^{*}$ et $q$ paratypes $\mathrm{n}^{\circ} 94292$, coparasites de Nippostrongylus sp.

HôTE : Iomys horsfieldi (Water house), $\mathrm{n}^{\circ} 94292$.

LOCALISATION : Intestin grêle.

Origine géographique: Kuala Langat Forest Reserve, Bukit Mandol Aborigine village, Klang, Selangor, Malaisie. Lat. $101^{\circ} 34^{\prime}$ N, Long. $2^{\circ} 55^{\prime}$.

\section{Description.}

Petits Nématodes enroulés de façon senestre, selon deux tours de spire, le long de leur ligne ventrale.

SYNLOPHE: il est de type Orientostrongylus et comporte 16 arêtes cuticulaires chez le mâle et $18 \mathrm{chez}$ la femelle (fig. 2, B). Les arêtes naissent sur le bord postérieur de la vésicule céphalique et s'étendent jusqu'en avant de la bourse caudale chez le mâle et de la vulve chez la femelle. 
MÂle: corps long de $1,7 \mathrm{~mm}$, large de $50 \mu$ dans sa partie moyenne. Vésicule céphalique haute de $40 \mu$ sur $20 \mu$ de large. Anneau nerveux, pore excréteur et deirides situés respectivement à $125 \mu, 185 \mu$ et $185 \mu$ de l'apex. Esophage long de $330 \mu$.

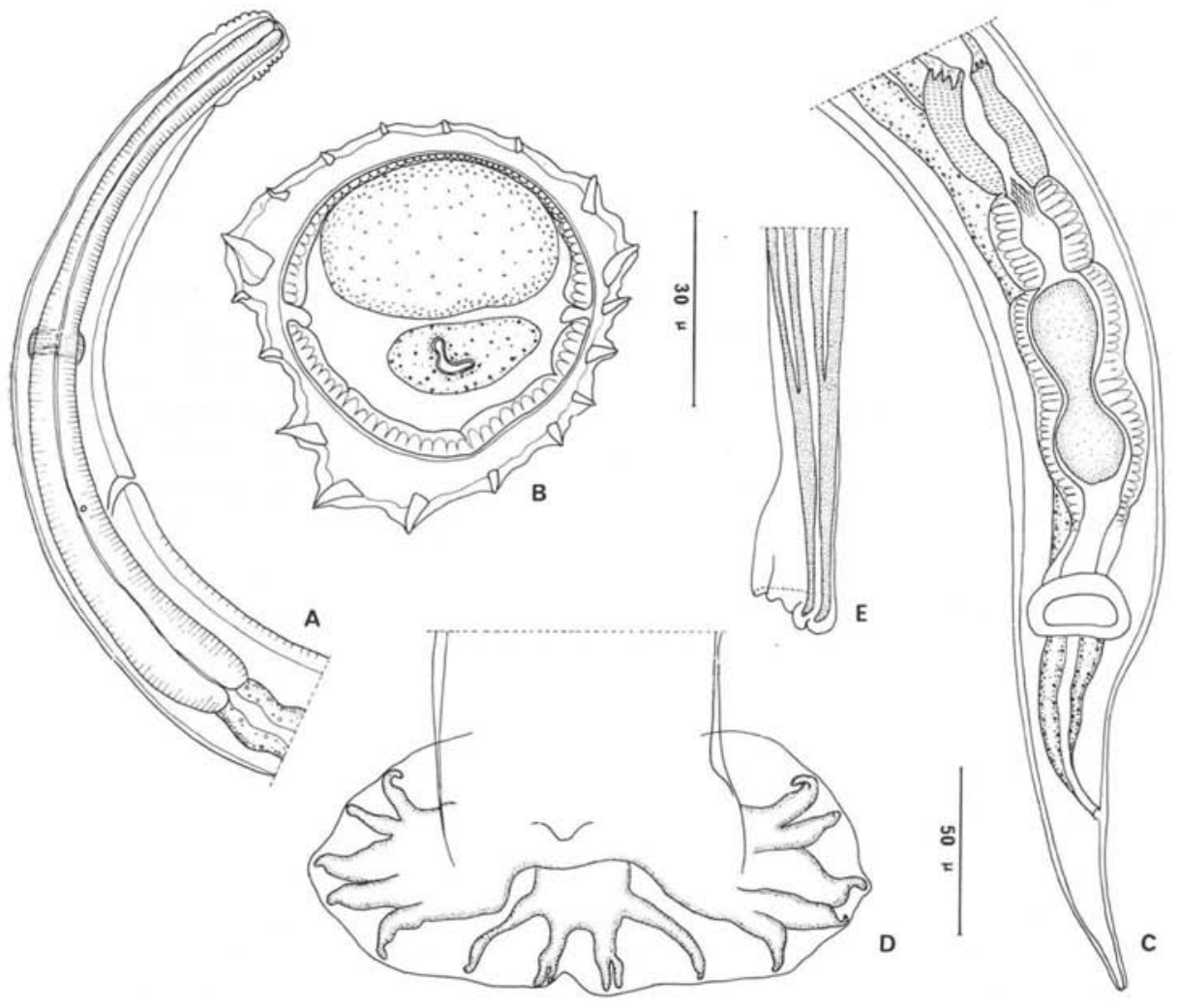

FIG. 2. - Orientostrongylus krishnansamyi n.sp. A, $\$$, extrémité antérieure, vue latérale droite. $\mathrm{B}, \hat{\delta}$, coupe transversale au milieu du corps. $\mathrm{C}$, + , extrémité postérieure, vue ventrale. $\mathrm{D}, \hat{\delta}$, bourse caudale, vue ventrale. $\mathrm{E}$, pointes des spicules disséqués. A, C, D, éch. : $50 \mu ; \mathrm{B}, \mathrm{E}$, éch. : $30 \mu$.

Bourse caudale sub-symétrique. Disposition des côtes bursales figurée en 2, D. Côte dorsale très épaisse, profondément divisée. Côtes 8 naissant juste en avant de la division de la côte dorsale (fig. 2, D).

Spicules longs de $290 \mu$. Leurs extrémités sont enfermées dans une membrane commune (fig. 2, E). Gubernaculum non vu. Cône génital très discret. 
Femelle : corps long de $2,2 \mathrm{~mm}$, large de $55 \mu$ dans sa partie moyenne. Vésicule céphalique haute de $32 \mu$ sur $20 \mu$ de large. Anneau nerveux, pore excréteur et deirides situés respectivement à $120 \mu, 170 \mu$ et $170 \mu$ de l'apex. Esophage long de $245 \mu$ (fig. 2, A).

La vulve s'ouvre à $105 \mu$ de la pointe caudale. Vagina vera, vestibule, sphincter et trompe longs respectivement de $30 \mu, 70 \mu, 29 \mu$ et $40 \mu$ (fig. 2, C). Utérus long de $250 \mu$ contenant 2 œufs hauts de $78 \mu$ sur $31 \mu$ de large.

Queue fine, longue de $55 \mu$ (fig. 2, C).

\section{Discussion.}

Cette espèce se range aisément dans le genre Orientostrongylus, dont elle possède tous les principaux caractères. Parmi les espèces du genre, $O$. tenorai est l'espèce la plus proche, car la disposition des côtes bursales est sensiblement identique, et, dans les deux cas, l'extrémité des spicules est enfermée dans une membrane. Cependant, chez $O$. tenorai, l'extrémité de chaque spicule est divisée en 2 pointes. De plus, le synlophe possède moins d'arêtes cuticulaires. Enfin, pour une longueur égale, l'utérus est deux fois plus court.

Nous pensons que nous pouvons séparer les spécimens de l'Iomys et nous les nommons Orientostrongylus krishnansamyi n. sp. en les dédiant à M. Krishnansamy.

\section{Remarques sur le genre Orientostrongylus}

La morphologie relativement primitive du genre Orientostrongylus avait conduit l'un de nous (cf. Durette-Desset, 1971) à le placer du point de vue phylétique à la base de la seconde branche évolutive de la lignée Heligmonella, cette lignée s'étant diversifiée chez les rongeurs d'apparition relativement récente, comme les Microtidés. et les Muridés.

Cependant, la place de ce genre restait aléatoire, car, bien que morphologiquement proche du genre Heligmonella, il n'était connu jusqu'à présent que chez des. Muridés et des Cricétidés orientaux. Sa présence chez un Tupaiidé, animal relique, est un élément qui nous semble venir confirmer notre hypothèse.

\section{REMERCIEMENTS.}

Ce travail a été effectué dans le cadre d'une mission en Malaisie à l'Institut de Recherche Médical de Kuala Lumpur. Nous exprimons nos vifs remerciements au $\mathrm{D}^{r}$ Bhagwan Singh, Directeur de cet Institut, pour nous avoir permis la réalisation de cette étude. Nous remercions également le personnel technique du laboratoire pour son aide efficace. 


\section{Bibliographie}

Durette-Desset (M.-C), 1970. - Caractères primitifs de certains Nématodes Héligmosomes, parasites de Muridés et de Cricétidés orientaux. Définition d'Orientostrongylus n. gen. Ann. Parasit. hum. comp., 45 (5), 829 à 837.

—, 1971. - Essai de classification des Nématodes Héligmosomes. Corrélations avec la paléobiogéographie des hôtes. Mém. Mus. nation, Hist. nat., A, Fr., 49, 126.

ERHARdova (B.), 1959. - Oswaldonema ryšavyi n. sp. und Viannella chinensis n. sp. (Nematoda: Heligmosomatidae) bei chinesischen nagern. Cěskoslovenska parasitologie, 6 (1), 93-96.

SINGH (K. S.), 1962. - Parasitological Survey of Kuman Region Part. XI. Four nematodes. from the Rat Rattus norvegicus. J. of Helm., 14 (2), 98-111. 